\title{
$\mathrm{CiSj}$
}

\section{AN ANTHROPOMORPHIC SPEECH PROCESSING BASED ON THE COCHLEAR MODEL AND ITS APPLICATION FOR CODING TASK*}

\author{
Petrovsky A.A. ${ }^{1,2)}$, Likhachov D.S. ${ }^{1)}$, W.Wan ${ }^{1)}$ \\ 1) Belarusian State University of Informatics and Radioelectronics, Computer Engineering Department, 220027, Minsk, \\ P.Brovki st., 6 (Belarus), E-mail: den2000@tut.by \\ 2) Bialystok Technical University, Real-Time Systems Department, 15-351, Bialystok, ul. Wiejska 45A (Poland), E- \\ mail: palex@it.org.by
}

\begin{abstract}
According to antisymmetry of basilar membrane (BM) movements, a new mathematical model of cochlea is derived using viscous cochlear fluid theory, and then transformed into a digital cochlear model with bilinear transformation. The frequency responses are found to be quite consistent with the experimental data, especially the high frequency slope is much more improved. A new cochlear map and $3 \mathrm{~dB}$ bandwidth characteristics for cochlear filter banks are obtained and presented, which will make applications of cochlear model more quantitative and accurate. Due to simplicity of its structure and reality of its characteristics, it will be proved the model can be used easily in speech processing system.
\end{abstract}

Keywords: Cochlear model, basilar membrane, tuning cochlear filter, auditory model, sinusoidal modeling.

\section{INTRODUCTION}

As a central part of auditory system, cochlea acts electrically as a highly overlapped bandpass filter bank. New results are continuously given out and knowledge of cochlea is gradually accumulated, but the mathematical model of cochlea is far from satisfaction. H.B.Allen, et al. [1,2] used Green's function method to find a fluid pressure with assumption of inviscous cochlear fluid and finally acquired a two-dimensional cochlear model represented by an integration equation. The amplitude frequency response is not very satisfied, especially low frequency slope is not as sharp as desired, structure of the model appears complicated either.

Cochlear characteristics can be influenced by many factors, but BM vibration and cochlear fluid motion should be two main factors. In the past years, much work was focused on BM properties, such as nonlinearity and activity of BM vibration [3-5], but cochlear fluid properties were paid little attention. S.Koshigoe, et al.[6] did some researches on cochlear fluid viscosity, but the work was incomplete. A two-dimensional mathematical model of cochlear with viscous fluid motion was developed in $[7,8]$, but high frequency slope of model's amplitude frequency response is not as sharp as desired, meanwhile the time variable was not discretized either.

Based on our previous results, a new mathematical model of cochlea is developed and transformed into a digital form using bilinear transformation. The model looks much simpler structure and comes to be a typical bandpass filter. The amplitude frequency response of the model is quite consistent with the experimental data [9-11]. The cochlear map which relates the center frequency of cochlear filters with BM locations are acquired and presented in a mathematical formula, and $3 \mathrm{~dB}$ bandwidth characteristics which relate the $3 \mathrm{~dB}$ bandwidth of cochlear filters with BM locations are also formulated.

\section{COCHLEAR MODEL WITH CONTINUOUS TIME AND CONTINUOUS SPACE}

According to [7], viscous cochlear fluid gradient along the BM can be represented as:

* This work was supported in part by the Bialystok Technical University under the grant W/WI/3/03 


$$
\begin{aligned}
& \frac{\partial p}{\partial x}=- \rho\left[\frac{\partial u_{S}}{\partial t}+\gamma\left(\frac{\pi}{H}\right)^{2} u_{S}\right] \times \\
& \times\left[1-\frac{4 \pi}{H^{2}} \sum_{i=1}^{\infty} \frac{\sin \left(\frac{(2 i-1) \pi x}{2 L}\right)}{\lambda_{i}^{2}(2 i-1)^{2}}\right], \\
& \lambda_{i}^{2}=\left[(2 i-1) \frac{\pi}{2 L}\right]^{2}+\left(\frac{\pi}{H}\right)^{2} .
\end{aligned}
$$

where $\rho$ and $\gamma$ denote fluid density and fluid viscosity, $L$ and $H$ denote BM length and half height, $p$ and $u_{s}$ denote fluid pressure and stapes velocity, and $x$ and $t$ represent spatial coordinate (from base to apex along the BM) and time coordinate respectively.

Due to the antisymmetry of BM movement in vertical direction $[2,12]$, it can be assumed that fluid pressure zero in helicortema, i.e.

$$
\left.p(x, y, t)\right|_{x=L}=0 \text {, }
$$

where $y$ is spatial coordinate vertical to BM.

Integrating (1) with boundary condition (2), we can obtain a fluid pressure distribution as follows:

$$
\begin{aligned}
p=- & \rho\left[\frac{\partial u_{S}}{\partial t}+\gamma\left(\frac{\pi}{H}\right)^{2} u_{S}\right] \times \\
& \times\left[x-L+\frac{8 L}{H^{2}} \sum_{i=1}^{\infty} \frac{\cos \left(\frac{(2 i-1) \pi x}{2 L}\right)}{\lambda_{i}^{2}(2 i-1)^{2}}\right] .
\end{aligned}
$$

In reference [7], (1) was derived with the assumption $\quad u_{s}=A \sin (\omega t+\varphi) \sin (\pi y / H)$, i.e. input signal was assumed to be sinusoid signal. Because speech signal can be represented with a finite sinusoid series [13] (it is obvious that results given here are also applicable to any other kinds of signals which can be represented by a finite sinusoid series) and (1) was obtained by solving a linear partial differential equation [7], (3) is applicable to speech signal according $t_{0}$ linear superposition.

From [14], it is known the following relation exists:

$$
p(x, t)=-\frac{1}{2}\left[\begin{array}{l}
k(x) z(x, t)+r(x) \frac{\partial z(x, t)}{\partial t}+ \\
+m(x) \frac{\partial^{2} z(x, t)}{\partial t^{2}}
\end{array}\right]
$$

where $p(x, t)$ denotes fluid pressure on the BM and $z(x, t) \mathrm{BM}$ vertical displacement, $k(x), r(x)$ and $m(x)$ represent stiffness, damping and mass of BM respectively. Combining (3) with (4), we can get a continuous space and continuous time cochlear model as follows:

$$
\begin{aligned}
& k(x) z(x, t)+r(x) \frac{\partial z(x, t)}{\partial t}+m(x) \frac{\partial^{2} z(x, t)}{\partial t^{2}}= \\
& =2 \rho\left[\frac{\partial u_{S}}{\partial t}+\gamma\left(\frac{\pi}{H}\right)^{2} u_{s}\right] \times \\
& \times\left[x-L+\frac{8 L}{H^{2}} \sum_{i=1}^{\infty} \frac{\cos \left(\frac{(2 i-1) \pi x}{2 L}\right)}{\lambda_{i}^{2}(2 i-1)}\right] .
\end{aligned}
$$

\section{COCHLEAR MODEL WITH DISCRETE TIME AND DISCRETE SPACE}

In eq(5), let:

$$
F(x)=2 \rho\left[x-L+\frac{8 L}{H^{2}} \sum_{i=1}^{\infty} \frac{\cos \left(\frac{(2 i-1) \pi x}{2 L}\right)}{\lambda_{i}^{2}(2 i-1)^{2}}\right] .
$$

then it follows

$$
\begin{gathered}
k(x) z(x, t)+r(x) \frac{\partial z(x, t)}{\partial t}+m(x) \frac{\partial^{2} z(x, t)}{\partial t^{2}}= \\
=\left(\alpha u_{S}+\frac{\partial u_{S}}{\partial t}\right) F(x) .
\end{gathered}
$$

We first discretize space coordinate in (7). To do so, let $x=k \Delta x$, where $0 \leq k \leq M$ and $x=L / M$, $M$ is the number of BM segments after discretization. (7) is transformed into the following form after discretization:

$$
\begin{aligned}
k_{k} z_{k}(t)+ & r_{k} \frac{d z_{k}(t)}{d t}+m_{k} \frac{d^{2} z_{k}(t)}{d t^{2}}= \\
& =\left(\alpha u_{s}+\frac{\partial u_{s}}{\partial t}\right) F_{k} .
\end{aligned}
$$

Transfer function of (8) is 


$$
H_{k}(s)=\frac{F_{k}(s+\alpha)}{k_{k}+r_{k} s+m_{k} s^{2}} .
$$

If bilinear transformation is applied to (9), i.e. let

$$
s=\frac{2}{T} \frac{1-z^{-1}}{1+z^{-1}}
$$

where $T$ denotes sampling interval, then transfer function for discrete space and discrete time cochlear model can be acquired

$$
\begin{aligned}
& \hat{H}(z)=\left[\begin{array}{l}
\left(\alpha T^{2}+2 T\right)+2 \alpha T^{2} z^{-1}+ \\
+\left(\alpha T^{2}-2 T\right) z^{-2}
\end{array}\right] F_{k} / \\
& {\left[\begin{array}{l}
\left(k_{k} T^{2}+2 r_{k} T+4 m_{k}\right)+ \\
+\left(2 k_{k} T^{2}-8 m_{k}\right) z^{-1}+ \\
+\left(k_{k} T^{2}-2 r_{k} T+4 m_{k}\right) z^{-2}
\end{array}\right]}
\end{aligned}
$$

With respect to speech signal, sampling frequency is generally no less than $8 \mathrm{kHz}$, i.e. $T \leq 1.25^{*} 10^{-4}$, the value of $\alpha$ is also no more than 10 (refer to Appendix), so all of the items with $T^{2}$ in the numerator of (11) can be omitted, and the following transfer function can be got

$$
\begin{aligned}
& \hat{H}(z)=2 T F_{k}\left(1-z^{-2}\right) / \\
& \left(\begin{array}{l}
\left(k_{k} T^{2}+2 r_{k} T+4 m_{k}\right)+ \\
+\left(2 k_{k} T^{2}-8 m_{k}\right) z^{-1}+ \\
+\left(k_{k} T^{2}-2 r_{k} T+4 m_{k}\right) z^{-2}
\end{array}\right)
\end{aligned}
$$

It can also be put into a much simpler form as follows

$$
\hat{H}(z)=A_{k} \frac{a_{0 k}\left(1-z^{-2}\right)}{1+b_{1 k} z^{-1}+b_{2 k} z^{-2}},
$$

where

$$
\begin{gathered}
A_{k}=\frac{2 T F_{k}}{a_{0} a_{0 k}}, a_{0 k}=\frac{1-b_{2 k}}{2}, \\
b_{i k}=b_{k} / a_{k}, b_{2 k}=c_{k} / a_{k}, \\
a_{k}=k_{k} T^{2}+2 r_{k} T+4 m_{k}, b_{k}=2 k_{k} T^{2}-8 m_{k}, \\
c_{k}=k_{k} T^{2}-2 r_{k} T+4 m_{k} .
\end{gathered}
$$

Note that (13) is actually a transfer function of a bandpass digital filter. From (13), we can get amplitude frequency response (Fig.1) and phase frequency response (Fig.2) for the cochlear model.

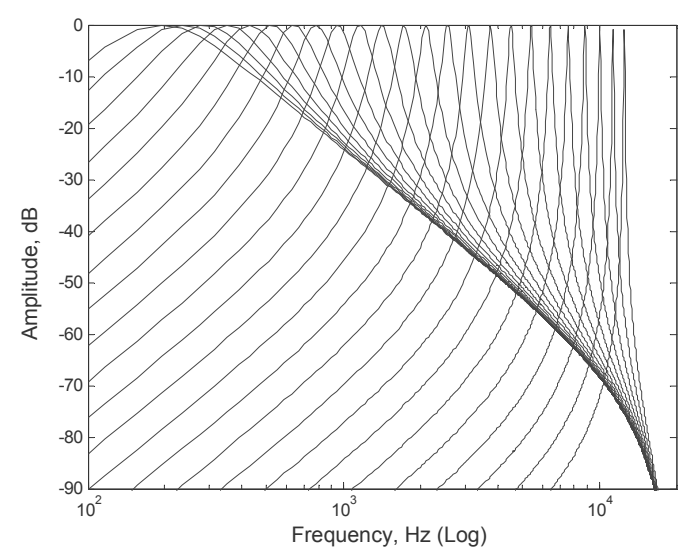

Fig.1. - Amplitude frequency responses of cochlear model for 24 equally spaced points on the BM $(0.6 \mathrm{~cm}$ to $3.6 \mathrm{~cm}$ from base to apex)

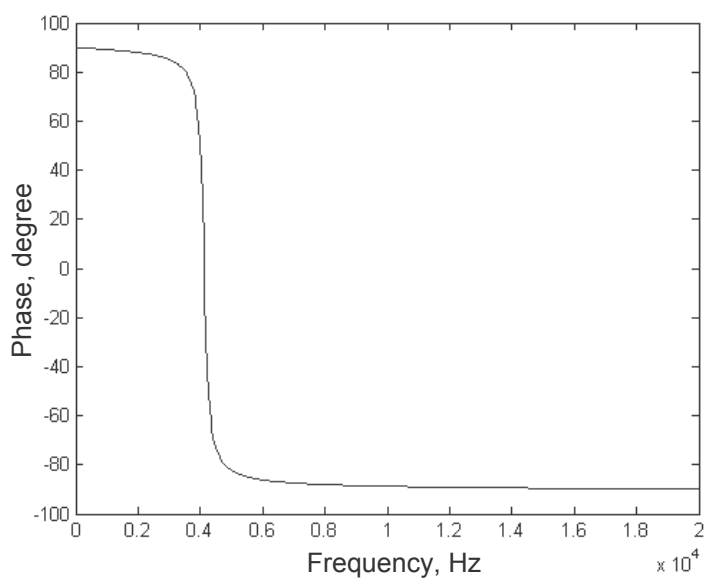

Fig.2. - Phase frequency response of cochlear model for a location near to BM apex (approximately $1.7 \mathrm{~cm}$ from the base)

\section{COCHLEAR MAP AND 3 DB BANDWIDTH CHARACTERISTICS}

From [15], we can get the following transfer function for an analog bandpass filter:

$$
\begin{aligned}
H(s) & =\frac{\left(\Omega_{0} / Q\right) s}{s^{2}+\left(\Omega_{0} / Q\right) s+\Omega_{0}^{2}}= \\
& =\frac{\Delta \Omega s}{s^{2}+\Delta \Omega s+\Omega_{0}^{2}},
\end{aligned}
$$

where $\Omega_{0}$ is angular central frequency of analog bandpass filter, and $\Delta \Omega$ is $3 \mathrm{~dB}$ bandwidth of the filter. If bilinear transformation (10) is applied to (14) (note the following relation exists in bilinear transformation 


$$
\Omega=\frac{2}{T} \operatorname{tg} \frac{\omega T}{2} .
$$

where $\omega$ denotes angular frequency of digital filter), then (14) can be changed into

$$
H(z)=a_{0} \frac{1-z^{-2}}{1+b_{1} z^{-1}+b_{2} z^{-2}},
$$

where

$$
\begin{aligned}
& a_{0}=\frac{\sin \omega_{0} T}{2 Q+\sin \omega_{0} T}, \\
& b_{1}=-\frac{4 Q \cos \omega_{0} T}{2 Q+\sin \omega_{0} T}, \\
& b_{2}=\frac{2 Q-\sin \omega_{0} T}{2 Q+\sin \omega_{0} T} .
\end{aligned}
$$

Also: $Q=\Omega_{0} / \Delta \Omega$ and $\omega_{0}=2 \pi f_{0}$, where $f_{0}$ is central frequency of digital filter in $\mathrm{Hz}$.

In bilinear transformation, if $\Delta \omega_{0}$ is small around the central frequency $\omega_{0}$, then relation between $\Omega$ and $\omega$ can be considered to be linear, so the following formula can be obtained from (15):

$$
\frac{\Delta \Omega}{\Delta \omega}=\left.\frac{\partial \Omega}{\partial \omega}\right|_{\omega=\omega_{0}}=\sec ^{2} \frac{\omega_{0} T}{2}, \sec x=\frac{1}{\cos x} .
$$

Let

$$
q=\omega_{0} / \Delta \omega
$$

then from (15) and (18), we can get the following relation

$$
\frac{Q}{q}=\frac{\sin \omega_{0} T}{\omega_{0} T} .
$$

Substituting (20) into (17) and using (19), we can get [16]

$$
\begin{gathered}
a_{0}=\frac{\Delta \omega T}{2+\Delta \omega T}, \\
b_{1}=-\frac{4 \cos \omega_{0} T}{2+\Delta \omega T}, \\
b_{2}=\frac{2-\Delta \omega T}{2+\Delta \omega T}
\end{gathered}
$$

Let $f=f / F_{S}=f T \quad\left(F_{S} \quad\right.$ is sampling frequency) which is a normalized frequency of called digital frequency, then $\omega T=2 \pi f T=2 \pi f=\omega$ which is digital angular frequency in radian, in this way, (21) can be changed to the following:

$$
a_{0}=\frac{\Delta \omega}{2+\Delta \omega},
$$

$$
\begin{gathered}
b_{1}=-\frac{4 \cos \omega_{0}}{2+\Delta \omega}, \\
b_{2}=\frac{2-\Delta \omega}{2+\Delta \omega} .
\end{gathered}
$$

It can be found there are only two independent variables in (22) because $b_{2}=1-2 a_{0}$.

Comparing (13) with (16), it is not difficult to find cochlear map and $3 \mathrm{~dB}$ bandwidth characteristics as follows

$$
\begin{aligned}
& \cos \omega_{0 k}=\frac{-b_{1 k}}{1+b_{2 k}}, \\
& \Delta \omega_{0 k}=\frac{2\left(1-b_{2 k}\right)}{\left(1+b_{2 k}\right)},
\end{aligned}
$$

where $\omega_{0 k}$ denotes central angular frequency for $k$ th cochlear filter.

Based on (23) and (24), cochlear map and $3 \mathrm{~dB}$ bandwidth characteristics can be calculated, and they are presented in Fig. 3 and Fig. 4 respectively.

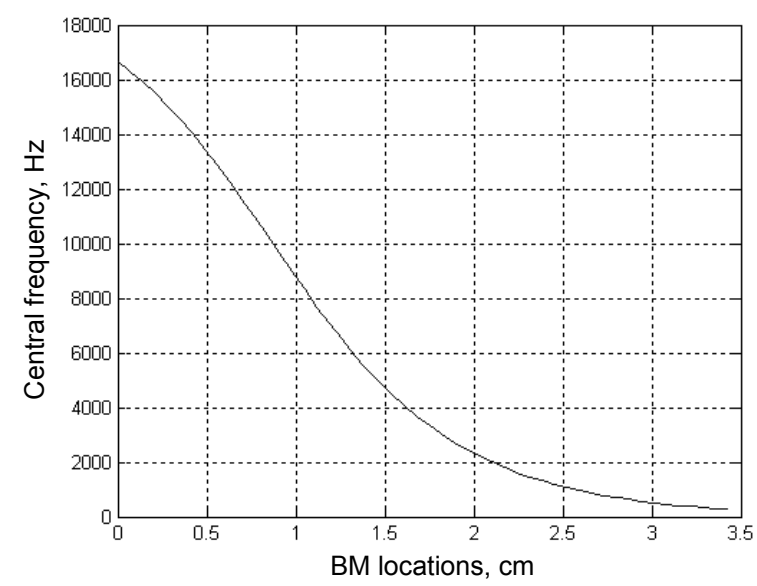

Fig.3. - Cochlear map (vertical coordinate denotes the central frequency of cochlear filters, and horizontal coordinate denotes BM locations from base to apex which have a biggest displacement)

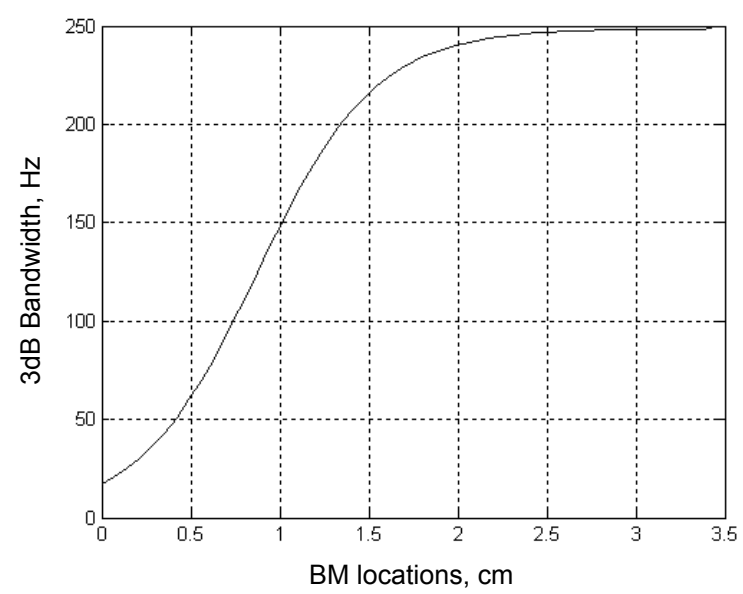

Fig.4. - 3 dB bandwidth characteristics for cochlear filter banks. (Vertical coordinate denotes $3 \mathrm{~dB}$ bandwidth of cochlear filters, and horizontal coordinate denotes BM locations from base to apex 
which have a biggest displacement)

\section{TUNING COCHLEAR FILTER BANK}

Let value $g=2 \cos \omega_{0}$ we can express (22a)$(22 \mathrm{c})$ in the following form $[21,22]$ :

$$
\begin{gathered}
b_{1}=\left(a_{0}-1\right) g \\
b_{2}=1-2 a_{0}
\end{gathered}
$$

So, transfer function for $k$-th cochlear filter can be described by following function:

$$
\oiint_{k}(z)=\frac{a_{0 k}\left(1-z^{-2}\right)}{1+\left(a_{0 k}-1\right) g_{k} z^{-1}+\left(1-2 a_{0 k}\right) z^{-2}},
$$

where $a_{0 k}$ and $g_{k}$ - parameters, which define filter bandwidth and central frequency correspondingly.

For defined value $q$ and central frequency $\omega_{0 k}$ the coefficients are following:

$$
\begin{gathered}
a_{0 k}=\frac{\omega_{0 k}}{2 q+\omega_{0 k}} \\
g_{k}=2 \cos \omega_{0 k}
\end{gathered}
$$

For filter bandwidth $\Delta \omega_{k}$ and central frequency $\omega_{0 k}$ the coefficients are:

$$
\begin{aligned}
& a_{0 k}=\frac{\Delta \omega_{k}}{2+\Delta \omega_{k}} \\
& g_{k}=2 \cos \omega_{0 k} .
\end{aligned}
$$

A functioning of the $k$-th tunable cochlear filter can be described with following equations [23]:

$$
\begin{aligned}
& w(n)=x(n)-g_{k} v(n-1)+2 v(n-2), \\
& v(n)=a_{0 k} w(n)+g_{k} v(n-1)-v(n-2), \\
& y(n)=v(n)-v(n-2),
\end{aligned}
$$

where $x(n)$ - number of the input set, $y(n)$ - number of the output set. So, the calculation of each element of the output set requires two multiplication and five additions.

\section{DISCUSSION}

In Fig.1, BM is evenly divided into 300 segments $(M=300)$. If $\mathrm{BM}$ length is $L=3,5 \mathrm{~cm}$, then ten amplitude frequency responses in Fig.1, are associated with the BM locations whose first point is $0.6 \mathrm{~cm}$ from the base and the last point is $1.63 \mathrm{~cm}$ with the space interval being $0.12 \mathrm{~cm}$. The figures presented here look much more consistent with the experimental data [10] [11], especially the high frequency slope has obviously been improved. It can also be seen that there are some differences among the peaks of curves. With the reduction of central frequency, pear value is gradually reduced, on the other hand, the peak value of curves will be gradually reduced with the increment of distance of locations from the BM base, but those differences are very small according to the computation, especially for those curves which have a high central frequency, the differences can almost be unidentified. From the viewpoint of computation, coefficient $A_{k}$ of (13) for $A_{k}=F_{k} / r_{k}$ and $r_{k}$ is assumed to be constant. From (6), it can be found than $F_{k}$ will be gradually reduced with the increment of $x$ because there are only two items related to $x$, one is $x$ itself and the other is cosine, the latter will reduce nonlinearly with the increment of $x$, the reduction of cosine is faster than increment of $x$, especially when $\mathrm{x}$ becomes large. From the other viewpoint, it seems that $\mathrm{BM}$ displacement will reduce with reduction of excitement frequency (assume excitement amplitude is invariant). Because low frequency signals excite BM apex, so it has to travel a longer distance along the $\mathrm{BM}$ and degradation seems to take place.

In Fig.2, a phase frequency response is presented. It is associated with a point near to BM apex, approximately $3.3 \mathrm{~cm}$ from the base (stapes). It is almost a linear function in linear frequency scale. Cochlear map is defined to be a relation between excitement frequencies or central frequency of cochlear filter and BM location which has a biggest displacement, excitement with different frequency excites a biggest displacement in different BM locations. From Fig.3, we find that relation between excitement frequency and location with biggest displacement is not exactly linear, that is to say, for those cochlear filters equally spaced on the BM, the differences between their central frequencies are not equally spaced in a linear frequency coordinate.

Cochlear map is conventionally deduced from experimental data [17]-[20], here it is deduced directly from the mathematical model of cochlear, and the result is quite consistent with the experimental data.

$3 \mathrm{~dB}$ bandwidth characteristics are defined to be a relation between $3 \mathrm{~dB}$ bandwidth of cochlear filters and BM locations with the biggest displacement. From Fig.4, we can see that bandwidth of cochlear filters is almost increased 
linearly with the increment of distance from BM base to BM center, but from center to apex, bandwidth is almost invariant. It seems that we can use a filter banks with same bandwidth to process speech signal for speech signal excites BM locations from center to apex.

It is believed that this result will make the application of cochlear model more quantitative and accurate.

\section{AN EXAMPLE OF IMPLEMENTATION COCHLEAR MODEL FOR SPEECH CODING}

Speech analysis process can be represented as shown in Fig.5 [24]. The approach considered here involves main ideas from papers $[25,26]$. The human auditory system model is used for improving spectral peaks selection.

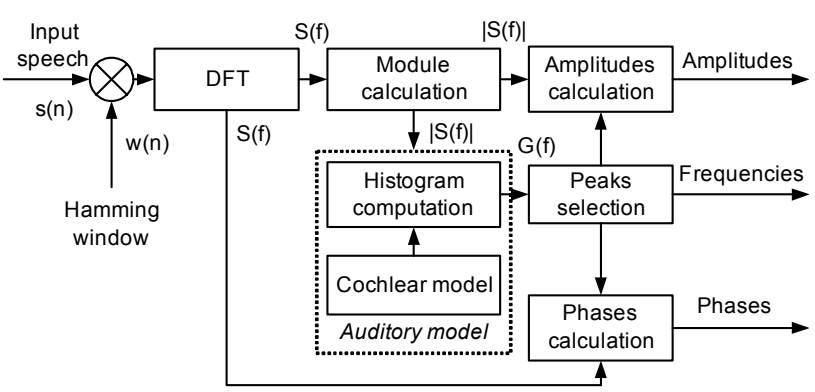

Fig.5. - Speech analysis scheme

Peak selection algorithm can be described with following steps:

Step 1. Array $D_{m}$ is calculated by following expression:

$$
\begin{gathered}
D_{m}\left(\operatorname{Ind}_{P}(l)\right)=\left|S\left(\operatorname{Ind}_{P}(l)\right)\right| \cdot H_{m}\left(\operatorname{Ind}_{P}(l)\right), \\
l=\overline{1, L_{P}}, \quad m=\overline{1, M},
\end{gathered}
$$

where $m$ - currently processed cochlear channel number; $M$ - a number of all cochlear filters; $L_{P}-$ a number of all spectral peaks; $\operatorname{Ind}_{P}(l)$ - array with indexes of obtained spectral peaks from array $|S(k)| ; \quad H_{m}\left(\operatorname{Ind}_{P}(l)\right) \quad-\quad$ amplitude-frequency response of the $m$-th cochlear filter in frequency position $\operatorname{Ind}_{P}(l), \quad l=\overline{1, L_{P}} ;\left|S\left(\operatorname{Ind}_{P}(l)\right)\right|-$ values of speech signal spectrum in frequency position $\operatorname{Ind}_{P}(l)$.

Step 2. Weighting coefficients $P_{m}^{l}$ for $l$-th spectral peak and $m$-th cochlear channel are calculated by following rule:

$$
\begin{gathered}
\text { if } D_{m}\left(\operatorname{Ind}_{P}(l)\right)>L v(i) \text { and } \\
D_{m}\left(\operatorname{Ind}_{P}(l)\right)<L v(i-1), i=\overline{1, N_{U}}
\end{gathered}
$$

$$
\text { then } P_{m}^{l}=2^{i} \text {, }
$$

where $N_{U}$ - a number of values in array $L v ; L v(i)$ is $i$-th element of level values array.

Step 3. Array histogram elements $G_{m}\left(\operatorname{Ind}_{P}(l)\right)$ for $l$-th spectral peak and $m$-th cochlear channel is computed by following formula:

$$
\begin{aligned}
& G_{m}\left(\operatorname{Ind}_{P}(l)\right)= \\
& =\left|S\left(\operatorname{Ind}_{P}(l)\right)\right| \cdot H_{m}\left(\operatorname{Ind}_{P}(l)\right) \cdot P_{m}^{l}=, \\
& \quad=D_{m}\left(\operatorname{Ind}_{P}(l)\right) \cdot P_{m}^{l}
\end{aligned}
$$

where $P_{m}^{l}$ - weighting coefficient for $l$-th spectral peak and $m$-th cochlear channel.

Step 4. Histogram $G\left(\operatorname{Ind}_{P}(l)\right)$ in position $\operatorname{Ind}_{P}(l)$ is computed by following expression:

$$
G\left(\operatorname{Ind}_{P}(l)\right)=\sum_{m=1}^{M} G_{m}\left(\operatorname{Ind}_{P}(l)\right), l=\overline{1, L_{P}},
$$

Step 5. $L$ spectral peaks are selected at frequencies that correspond to $L$ largest element values in the histogram $G\left(\operatorname{Ind}_{P}(l)\right)$.

An example of histogram $G$ for one speech signal frame is presented in Fig.6. X-direction frequencies from 0 to $4000 \mathrm{~Hz}$ (sampling frequency is $8000 \mathrm{~Hz}$ ). Y-direction - calculated weights (the bigger is an element histogram weight - the more important is its role in human speech recognition).

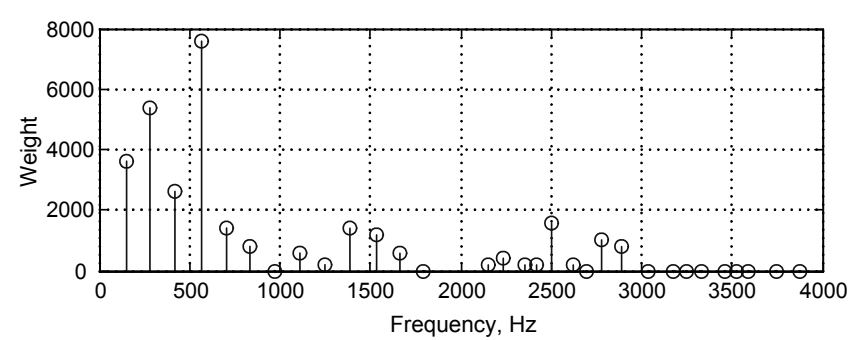

Fig.6. - A histogram for one speech signal frame

Selected spectral peak locations for one speech signal frame are presented in Fig.7 (red circles).

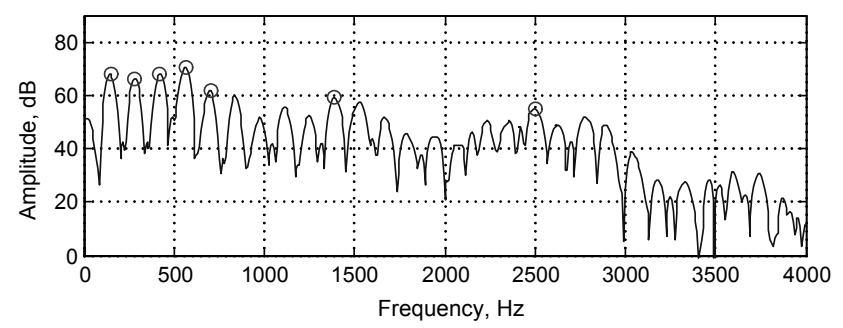

Fig.7. - Selected spectral peaks

Speech synthesis procedure presumes generating sinusoids according to frequency and phase 
parameters, weighed by amplitude and then summed to produce a frame of synthesized speech as shown in Fig.8. The resulting speech signal is formed by overlapping and adding each frame of the synthesized speech.

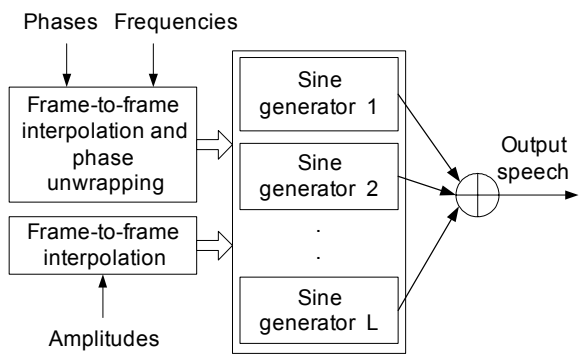

Fig.8. - Speech synthesis scheme

As an example a piece of the original speech signal in time domain is presented in Fig.9 (sampling frequency is $8000 \mathrm{~Hz}$, signal amplitude is 16 bits, male voice) and his spectrogram - in Fig.10.

A piece of the synthetic speech signal is presented in Fig. 11 and his spectrogram - in Fig.12.

Following parameters were used for speech signal analysis: length of the Fourier transform $N_{F}$ is 1024 , a number of all cochlear filters $M$ is 50 , a number of sinusoids is 7 ; analyzing window length is $32 \mathrm{~ms}$; frame length is $22,5 \mathrm{~ms}$.

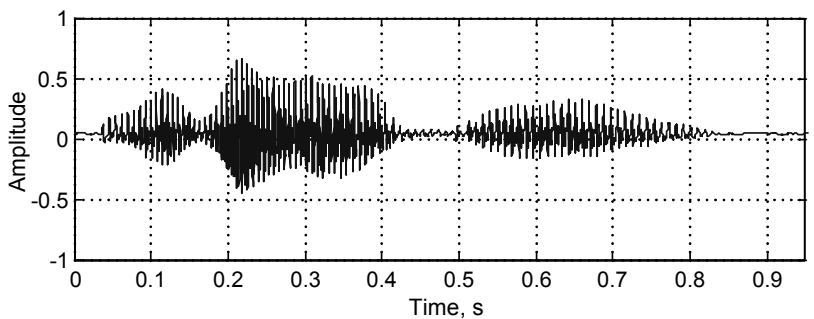

Fig.9. - The original speech signal

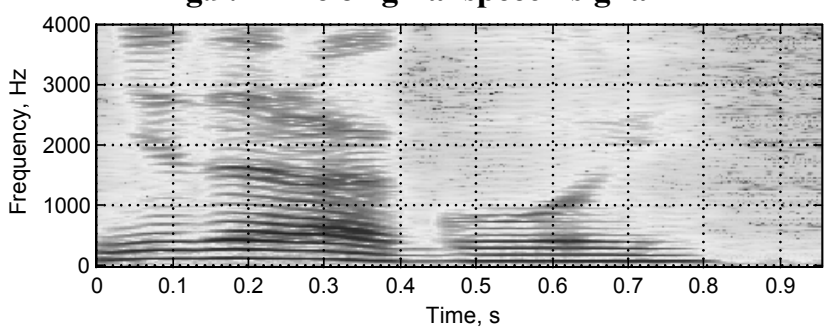

Fig.10. - The original speech signal spectrogram

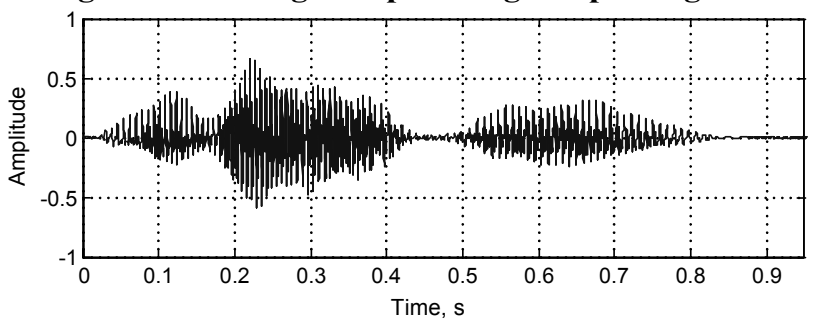

Fig.11. - The synthetic speech signal

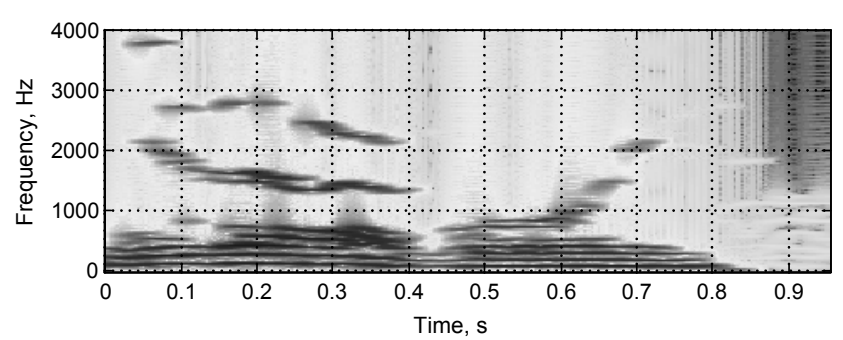

Fig.12. - The synthetic speech signal spectrogram

So, the experiment results show that the synthesized output signal retains most of the clearness of the original speech. It has good legibility and allows speaker recognition. Compared with the conventional speech coding systems, the proposed encoder has simple algorithmic realization and does not require making voice/unvoiced decision and pitch estimation during the speech analysis. Therefore it is less sensitive to a background noise and to changing a speaker than conventional speech encoders. Assuming that a vector quantization of speech parameters is used the average coding bit-rate is from 2 to $8 \mathrm{kbit} / \mathrm{s}$.

\section{CONCLUSION}

Based on previous cochlear mechanics put forward by the authors, a news cochlear model is obtained using both antisymmetry of BM movement and theory that speech signal can be represented by a finite sinusoid series. By using bilinear transformation, the model is changed into a secondorder digital cochlear model, that is to say, cochlea can be modeled with a second-order difference equation. The frequency slope is sharpened and thus looks more realistic.

According to characteristics of bilinear transformation, a new cochlear map and $3 \mathrm{~dB}$ bandwidth characteristics are acquired. The cochlear map is also quite consistent with the experimental data, except in the BM apex. The cochlear model put forward in this paper is believed to simple in structure, and therefore easy to apply, but it is far from complete and much work can be done because $\mathrm{BM}$ nonlinearity and other special properties of BM is not included, this model makes such inclusion feasible.

\section{REFERENCES}

[1] B.Allen, "Two-dimensional cochlear fluid model: new results", J.Acoust. Soc. Am. 61., 1971, pp.110-119.

[2] B.Allen, M.M.Sondhi, "Cochlear macromechanics: time domain solutions", J.Acoust. Soc. Am. 66., 1979, pp.123-132.

[3] R.Diependaal, "Nonlinear and active cochlear models: analysis and solution methods", Ph.D.thejsis, Delft University, 1988. 
[4] J.W.Matthews, "Mechanical modeling of nonlinear phenomena observed in the peripheral auditory system", Ph.D.thesis, Washington University, St.Louis, MD, 1980.

[5] S.T.Neely, D.O.Kim, "A model for active elements in cochlear biomechanics", J.Acoust. Soc. Am. 79, 1986, pp.1472-1480.

[6] S.Koshigoe, W.K.Kwok, and A.Tubis, "Effects of perilymph viscosity on low-frequency intracochlear pressures and the cochlear input impedance of the cat", J.Acoust. Soc. Am. 74, 1983, pp.486-492.

[7] W.G.Wan, C.X.Fan, "A new solution for cochlear macromechanics", Acustica 75, 1991, pp.79-82.

[8] W.G.Wan, C.X.Fan, "A new cochlear model: viscous fluid motion", J.Acoust. Soc. Am. 89, 1991, pp.1865.

[9] L.Robles, M.A.Ruggero, N.C.Rich, "Basilar membrane mechanics at the base of the chinchilla cochlea. I. Input-output functions, tuning curves and response phases", J.Acoust. Soc. Am. 80., 1986, pp.1364-1374.

[10] W.S.Rhode, "Observation of the vibration of the basilar membrane in squirrel monkeys using the Mossbauer technique", J.Accoust. Soc. Am. 49, 1971, pp.1218-1231.

[11] W.S.Rhode, L.Robles, "Evidence from Mossbauer experiments for nonlinear vibrations in the cochlea", J.Accoust. Soc. Am. 55, 1974, pp.588596.

[12] J.B.Allen, "Cochlear modeling", IEEE ASSP Magazine 1, 1985, pp.3-29.

[13] R.J.McAulay, T.F.Quatieni, "Speech analysis/synthesis based on a sinusoidal representations", IEEE Trans. on ASSP 34, 1986, pp.744-754.

[14] Monderer, "Exploring the space-time structure at the output of a cochlear model", Ph.D. thesis, Columbia University, New York, 1988.

[15] D.Johnson, J.Johnson, and H.Moore, A bandbook of active filters, Prentice-Hall, Inc. Englewood Gliffs, N.J., 1980.

[16] A.A.Petrovsky, "Methods and microprocessor means of processing broadband and fastflowing processes in real time", Nauka i Tekhnika, Minsk, 1988.

[17] S.Puria, J.B.Allen, "A parametric study of cochlear input impendance", J.Acoust. Soc. Am. 89, 1991, pp.287-309.

[18] D.H.Eldredge, J.D.Miller, and D.A.Bohne, "A frequency-position map for the chinchilla cochlea", J.Acoust. Soc. Am. 69, 1981, pp.10911095.

[19] D.Greenwood, "A cochlear frequencyposition function for several species -- 29 years later", J.Acoust. Soc. Am. 87, 1990, pp.2592-2605.
[20] M.C.Liberman, "The cochlear frequency map for the cat: Labeling auditory nerve fibers of known characteristics frequency", J.Acoust. Soc. Am. 72, 1982, pp.1441-1449.

[21] A.A.Petrovsky, J.A. Ganushkin. "Syntheses of digital bandpass filters. Radiotechnika", Radiotekh. Electronik, 1985, vol. 10, pp. 24-25, 1985.

[22] A.Petrovsky. "The synthesis of high order digital bandpass filter with tunable center frequency and bandwidth", VIII Europ. Sig. Proc. Conf. (EUSIPCO 96). Trieste, Italy, 10-13 September 1996, pp. 1527-1530.

[23] Alexander A. Petrovsky, Jaroslaw Baszun. "Design of Digital Tunable Bandpass Filters: Synthethis and Applications", Instytut Informatyki, Politechnika Bialostocka, Kwartalnik electroniki i telekomunikacji, 2001, pp. 5-25.

[24] Likhachov D.S., Petrovsky A.A. "Improved auditory-based speech coding using psychoacoustic model based on a cochlear filter bank and an average localized synchrony detection", CISIM'2003, pp.11-19.

[25] A. M. A. Ali et al., "Robust auditory-based speech processing using the average localized synchrony detection", IEEE Transactions on Speech and Audio Processing, vol.10, no.5, July 2002, pp.1447-1459.

[26] Oded Ghitza, "Auditory Models and Human Performance in Tasks Related to Speech Coding and Speech Recognition", IEEE Transactions on Speech and Audio Processing, vol.2, no.1, part II, 1994 - pp. 115-132.

Alexander Petrovsky received
the Dipl.-Ing. Degree in computer
engineering in 1975 from the Minsk
Radio-Engineering Institute, Minsk,
USSR. In 1980 he received the Ph.D.
degree from the Minsk Radio-
Engineering Institute, and in 1989 he
is the Doctor of Science from The

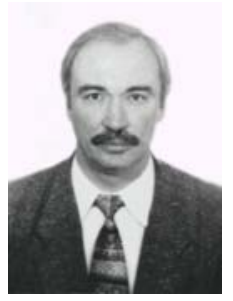
Institute of Simulation Problems in Power Engineering, Academy of Science, Kiev, USSR.

Since 1990, he has been Professor, head of the Computer Engineering department at the Belarusian State University of Informatics and Radioelectronics, Minsk, Belarus, and he is with Real-time department, Faculty of Computer Science, Bialystok Technical University, Poland. Recently his research interest are acoustic signal processing, such as speech and audio coding, noise reduction and acoustic echo cancellation, robust speech recognition, and real-time signal processing: design and implementation of signal processing systems. He is a member of AES, IEEE, IIAV 


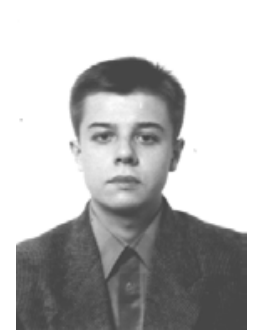

Denis Likhachov received the Engineering degree in Designing and Technology of Electronic Computing Facilities from the Belarusian State University of Informatics and Radioelectronics, Minsk, Belarus, in 2000.

Currently he is Assistant-professor and Ph.D.-student at the Computer Engineering Department, Belarusian State University of Informatics and Radioelectronics, Minsk, Belarus. His research interests include digital signal processing, auditory modeling and speech compression.

Professor Wanggen Wan graduated the Department of Information Engineering, Xidian University, Xian, Shaanxi, P.R.China. He was Assitant-professor at the Computer Engineering department of Belarusian State University of Informatics and Radioelectronics in 1993. Recently, he is with Department of Electrical and Electronic Engineering Hong Kong University of Science and Technology. His research interest in the field of speech processing. 